\title{
Exploratory Study of the Cardiac Dynamic Trajectory in the Embedding Space
}

\author{
Jorge Oliveira $^{1}$, Bruna Cardoso ${ }^{2}$, Miguel T Coimbra ${ }^{1}$ \\ ${ }^{1}$ Instituto de Telecomunicações, Faculdade de Ciências da Universidade do Porto, Portugal \\ ${ }^{2}$ Faculdade de Medicina, Faculdade de Ciências da Universidade do Porto, Portugal
}

\begin{abstract}
In this paper, the topological and dynamical properties of the heart sounds are assessed. The signal is preprocessed and projected into an embedding subspace, which is more suitable to detect the irregularities and the unstable trajectories registered during the cardiac murmurs than the original heart sound signal.

We present a method for heart murmur classification divided into five major steps: a) signal is divided into heart beats; b) entropy gradient envelogram is computed from the pre-processed signal; c) the orbital trajectories are reconstructed using the embedding theory; d) $n$ orbits in the embedding subspace are extracted (per heart beat); $e$ ) the median of the $n$ orbits is used as an input to K-Nearest Neighbors (KNN) classifier.

The experimental results achieved are in agreement with the current state of art for heart murmur classification.
\end{abstract}

\section{Introduction}

Heart sound auscultation using a traditional stethoscope is the simplest, fastest and cheapest method for heart examination. Although the importance of the traditional auscultation methods has decreased due to its inherent restrictions. The phonocardiogram (PCG) has preserved its importance in pediatric cardiology, cardiology, and internal diseases, evaluating congenital cardiac defects. The phonocardiogram is divided into heart cycle (S11) components such as: S1 (first heart sound) and S2 (second heart sound) [1]. These establish the boundaries of the other two fundamental components of a heart cycle: the systole (S21), and the diastole (S12). The S1 and S2 are generated by the opening and closing of the heart valves, in pathogenic situations additional sounds such as S3, S4 or murmurs are listened [1]. Heart murmurs are turbulence phenomena characterized by momentum diffusion, high momentum convection, and rapid variation of pressure and velocity both in space and time [1]. The automatic detection of heart murmurs strongly depends on the extraction of an appropriate set of features, which are hopefully capable of splitting the data into two or more categories: normal and different types of abnormal cases.

Ahlstrom proposed a set of 207 features composed by Shannon energy, wavelet coefficients, fractal dimensions and recurrence quantification analysis. A subset of 14 features was derived using a Pudil's sequential floating forward selection algorithm. Using a neural network classifier, this subset achieved $86 \%$ of accuracy [2]. Delgado-Trejos compared three types of features: spectral, perceptual and fractal features. Using a K-nearest neighbor's classifier they observed that fractal features provide the best accuracy $(97,17 \%)$ followed by spectral (95, 28\%) and perceptual features (88,7\%). This fact it is explained by the presence of long-range (fractal) correlation along with distinct classes of non-linear interactions [3]. The feature set described in our previous work [4] is a combination of time-frequency domain, perceptual and fractal analysis. We also proposed: 1) the dimension correlation curve; 2 ) the exponential decay of the false nearest neighbor integral as new features. These express the higher complexity registered for the murmur cases when compared to the normal cases and the nonexistence (mostly of the times) of a stable plateau in dimension correlation curve for the murmur cases [5]. This motivate us to continue our study on the topological properties of the heart sound signals. The two main contributions presented in this paper are: 1) a new embedding subspace, where orbital trajectories are associated to heart sounds; 2 ) from the orbital trajectories, a new topological feature is extracted, namely $\mathrm{n}$ orbits.

The paper is organized as follows: in the second section, the methodology is explained. In the third sections, the materials are presented. In the fourth and fifth sections, results are presented and conclusions are withdrawn.

\section{Methods}

\subsection{Signal pre-processing}

In order to attenuate noise, the PCG signal $x(t)$ is filtered with a band-pass, zero phase Butterworth filter order $10(30-450 \mathrm{~Hz})$. Afterwards, the mean $\bar{x}$ is subtracted 
from the signal $x(t)$ and it is scaled as $X_{N}(t)=$ $\frac{x(t)-\bar{x}}{\max \left(x\left(t^{\prime}\right)-\bar{x}, t^{\prime} \in[1, M]\right)}$, where $M$ is the time series length.

\subsection{Entropy gradient}

To compute the entropy gradient, we first convert $X_{N}$ into a sequence of delay vectors as it is described by the embedding theorem [6]. In more detail, we wrap the original signal around a cylinder as it is shown in Figure 1. This cylinder is not static and it moves one or more samples per unit of time to the right ${ }^{1}$. It is advisable to tune the cylinder speed depending to the sampling rate of the signal. In our case, the cylinder moves 40 samples per unit of time. From this cylinder, a sequence of $P=M-$ $\Gamma(m-1)$ delay vectors are collected sequentially, where $M$ is time series length, $\Gamma$ is the time lag and $m$ is the vector dimension [6]. For example, the $i^{\text {th }}$ delay vector $d^{i}$ is collected after having shifted $j^{\text {th }}$ units of time to the right as:

$$
d_{j}^{i}=\left\{X_{N}([i+j] \% M), \ldots, X_{N}([i+j+(m-\right.
$$

1) $\Gamma] \% M)\} \in \mathbb{R}^{m}, i \subset\{1, \cdots, P\}, j \subset\{1, \cdots, M\}^{2}$

In order to generate entropy fluctuations over the time, a set of data points are not assembled into delay vectors and they belong to so called "rejection region", in our case $X_{N}^{r e j}=X_{N}(P+1), \cdots, X_{N}(M)$. This region is not taken into account for the total entropy of the system. Finally, all delay vectors are normalized $\left(\tilde{d}_{j}^{i}=\frac{d_{j}^{i}}{\sqrt{P}}\right)$ and stored into the matrix $K_{j}$.

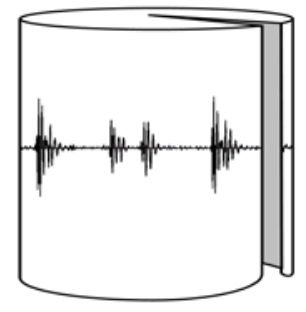

Figure 1. An infinite PCG signal, obtained by 'wrapping' a finite one around a cylinder. New samples can be generated by simply rotating the cylinder to the left or to the right. The rejection region is represented in gray.

The autocorrelation of each embedding matrix $K_{j}$ is computed as:

$$
C_{j}=K_{j}^{T} K_{j}, \quad C_{j} \in \mathbb{R}^{(m \times m)}
$$

\footnotetext{
${ }^{1}$ Shifting the cylinder to the left gives equivalent results.
}

The $C_{j}$ matrix is a metric of correlation between delay vectors in the time series $X_{N}(t) . C_{j}$ is a real symmetric matrix, its eigenvalues are real and its eigenvectors are orthogonal. Let $D_{j}$ be the diagonal matrix of the eigen values of $C_{j}$ sorted in a descending order $\lambda_{1}>\lambda_{2}>\ldots>\lambda_{m}$. The highest eigenvalues are associated with the most relevant structures of the signal. In contrast, the lowest eigenvalues are usually associated with very small variations and noise [7]. Using the $D_{j}$ vector, the $j^{t H}$ entropy gradient is given by:

$$
\nabla_{\text {entropy }}(j)=\sum_{k=1}^{m} \hat{\lambda}_{j}^{k} \log _{2}\left(\hat{\lambda}_{j}^{k}\right)
$$

Where $\hat{\lambda}_{j}^{k}$ is the normalized eigenvalue $\lambda_{j}^{k}$. The results of applying the $\nabla_{\text {entropy }}$ to a PCG signal are shown in Figure 2.

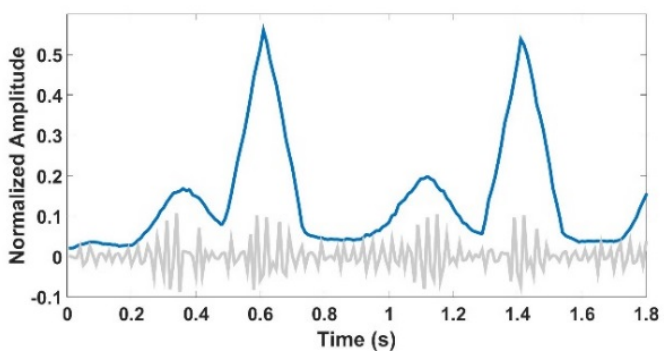

Figure 2. The $\nabla_{\text {entropy }}$ (in blue) and the normalized PCG signal (in gray).

It is important to understand, that a simply cylinder rotation to the left or to the right causes parts of the signal to move in and out of this "rejection" region $X_{N}^{r e j}$. As a consequence, the fluctuations in the total entropy are caused by different degrees of predictability over time. We expect that when noisy parts of the signal enter and leave $X_{N}^{r e j}$, they will not generate significant entropy variations. But if noisy parts of the signal enter on $X_{N}^{r e j}$ and signal parts leave it, this is quite likely to create a significant amount of entropy variation. In our experimental setups, the entropy gradient is generated by setting $(m=5, \Gamma=100)$.

\subsection{Embedding space}

After the $\nabla_{\text {entropy }}$ is computed, delay vectors $(m=2, \Gamma=$ 2 ) are extracted sequentially and saved into the embedding matrix $L$.

\footnotetext{
$2 \%$ is the module operator
} 

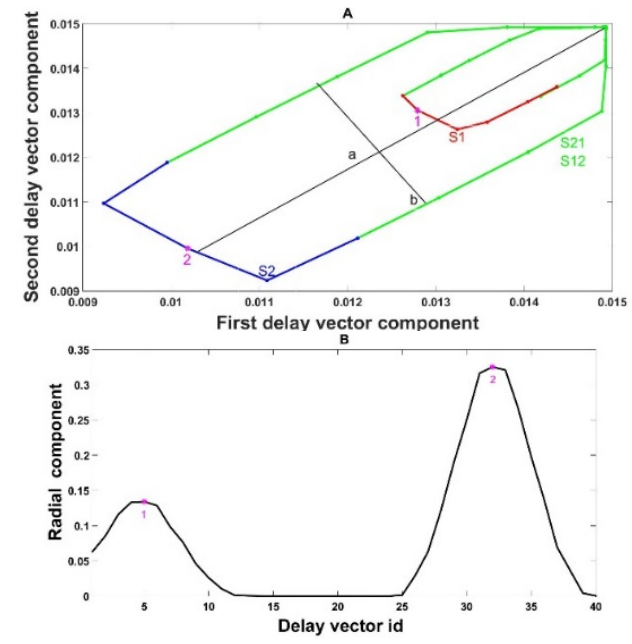

Figure 3. (A) Orbital trajectories in the embedding subspace belonging to a healthy person during a single heart cycle. (B) Radial component of each delay vector. ${ }^{3}$

The orbital trajectories in the embedding space are created from the embedding matrix $L$, where each delay vector $d_{x, y}=\left(d_{x}, d_{y}\right)$ is directly mapped into point in such a space. In each heart cycle for normal individuals, it is possible to distinguish two orbits: the S1 and S2. The S2 is generated by the closing of the semilunar valves. This mechanism is strongly dependent on external and internal cardiac factors, like the blood pressure in the aortic and pulmonary arteries and also the blood pressure in the ventricles [1]. S1 is a less complex phenomenon, generated by the A-V valves closing, which uniquely depends on internal cardiac factors like the blood pressure in the atriums and ventricles [1]. Therefore we speculate that $\mathrm{S} 1$ should be more predictable than S2, given that it does not depend on so many factors. Since entropy gradient measures the predictability degree of different sources, if our speculations are true, then the S1 trajectories (more predictable, lower entropy gradient) should be typically bounded and contained inside of the S2 trajectories (less predictable, higher entropy gradient). More experiments are required to verify this hypothesis but an example of this is beautifully depicted in Figure 3A, corresponding to a real heart sound cycle collected from a healthy person.

The heart sound trajectories are usually eccentric (see Figure $3 \mathrm{~A}$ and $3 \mathrm{~B}$ ), and their two main axis encode interesting information. The major axis $\boldsymbol{a}$ gives us the maximum entropy gradient variation within the whole signal, which can be interpreted as a useful measure of the unpredictability strength of the signal. The minor axis $\mathbf{b}$ is less informative, proportional to the temporal duration.

\footnotetext{
${ }^{3}$ https://youtu.be/FC5PsWtdvI4

${ }^{4}$ https://youtu.be/aJvNtba-vIY
}
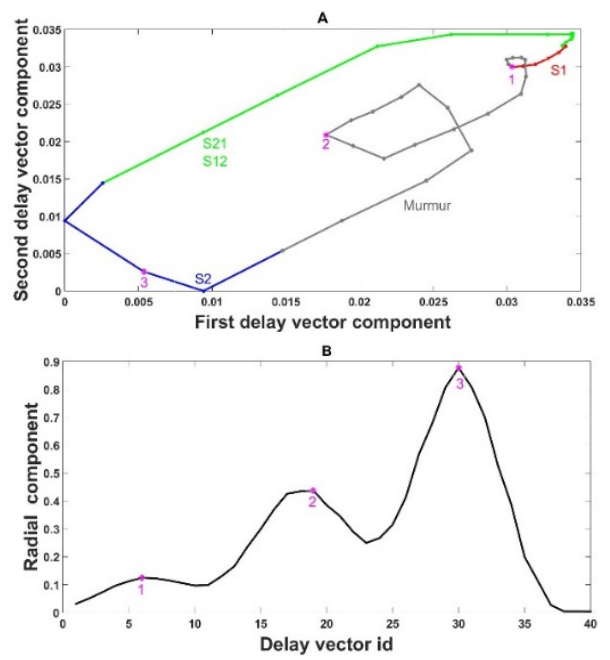

Figure 4. (A) Orbital trajectories in the embedding subspace belonging to an individual with heart murmur during a single heart cycle. (B) Radial component of each delay vector. ${ }^{4}$

In contrast, individuals with heart murmurs, revealed more complex and unstable trajectories. In Figure 4A, it is possible to observe a systolic murmur phenomenon, where the existing murmur is partially overlapping the S1 and S2 components. This murmur is responsible for the destruction of the topological structures which existed in the heart sound signal. This particular murmur generates swirls, which are usually associated to the turbulence phenomenon generated by a leakage of blood backwards through the Tricuspid or Mitral valve, each time the right or the left ventricle contracts respectively [1]. Because of the swirls, the number of orbits detected compared to the healthy cases are in general different, see Figures $3 \mathrm{~B}$ and 4B. This feature is going to be used to distinguish healthy and ill subjects.

\subsection{Downsampling $\mathrm{L}$}

In order to speed up our computation and to reduce the redundancy in the embedding matrix $L$ a downsampling methodology is implemented: 1) From each pair of delay vectors $\left(\forall d_{i}, d_{j,}\right) \in L$ and $L=\Re^{P \times 2}$, the Euclidean distance is computed and saved into the matrix $H=\Re^{P \times P}$; 2) set the $\operatorname{diag}(H)=\infty$, since the auto-distance is always the minimum distance when compared to any other point; 3) For $\forall j$ in $H_{, j}$ select the closest delay vector $\overline{\bar{d}}_{l}=$ $\left.\min \left(\forall j \in\{1, \ldots, l\}, i \neq j:\left|H_{i . j}\right|\right) ; 4\right)$ the delay vector $j$ is 
discarded if exists a neighbor delay vector $\left(\mathrm{d} \_\mathrm{i}\right)^{\overline{ }}$ closer than a pre-defined threshold (in our case $\leq \llbracket 10 \bar{\rrbracket} \wedge(-8)$ ).

\subsection{Feature Extraction}

From each heartbeat, $n$ distinct trajectories in the embedding space $L$ are computed by: 1) convert each delay vector $d_{x, y}$ in $L$ into the polar coordinate system $d_{r, \theta} ; 2$ ) Extract the radial component of each delay vector and store it into a vector $F_{r}$; 3) compute $G_{r}=1-\frac{F_{r}}{\max \left(F_{r}\right)}$; 4) select local maximums in $G_{r}$ which satisfies an optimal criterium (in the present case, a local maximum is selected if the peak prominence is $>0.05$ ) see Figures $3 \mathrm{~B}$ and 4B; 4) count the number of extracted peaks. Finally, the median of all heartbeats is used as feature for classification [8].

\section{Materials}

The heart sounds used have been collected in Real Hospital Português (Recife, Brasil) [9]. The auscultation spots (aortic-AV, pulmonary-PV, tricuspid-TV and mitralMV) were auscultate sequentially for about 15 seconds at $4000 \mathrm{~Hz}$ sampling frequency. Two cardiacpulmonologists manually annotated the locations of S1 and S2 using the Audacity software. The dataset is compose by (7-TV, 8AV 10-PV, 9-MV) normal cases and (7-TV, 8-AV, 10-PV, 9-MV) systolic murmur cases.

\section{Results}

\subsection{Experimental setup}

The number of orbits is used for training and testing a $K$ Nearest Neighbors (KNN) algorithm with $K=3$. Since the size of the dataset is relatively small, a leave-one-out cross validation methodology was implemented. This procedure is repeated for the mitral, tricuspid, aortic and pulmonic auscultation focus independently, the results are shown in Table I. The confidence intervals are computed using a ttest with $95 \%$ confidence level.

MURMUR CLASSIFICATION RESULTS IN DIFFERENTS AUSCULTATION FOCUS

\begin{tabular}{|c|c|c|c|c|}
\hline \multirow{2}{*}{$\begin{array}{c}\text { Results } \\
\text { (\%) }\end{array}$} & \multicolumn{4}{|c|}{ Feature set containing all features } \\
\cline { 2 - 5 } Recall & $\boldsymbol{M V}$ & $\boldsymbol{T V}$ & $\boldsymbol{A V}$ & $\boldsymbol{P V}$ \\
\hline Precision & $0.70-1$ & $0.78-1$ & $0.75-0.75$ & $0.73-0.78$ \\
\hline Sensitivity & $0.67-1$ & $0.71-1$ & $0.75-0.75$ & $0.70-0.80$ \\
\hline Specificity & $0.70-1$ & $0.78-1$ & $0.75-0.75$ & $0.73-0.78$ \\
\hline F-Measure & $0.70-1$ & $0.78-1$ & $0.75-0.75$ & $0.73-0.78$ \\
\hline Accuracy & $0.81-0.80$ & $0.83-088$ & $0.77-0.84$ & $0.74-0.76$ \\
\hline Cohen's kappa & $0.64-0.64$ & $0.86-0.86$ & $0.75-0.75$ & $0.75-0.75$ \\
\hline & & $0.71-0.71$ & $0.50-0.50$ & $0.50-0.50$ \\
\hline
\end{tabular}

The proposed feature does not perform equally in each auscultation spot. The best results were achieved in the Tricuspid spot, mostly because, the murmurs are well audible in this spot (leaky in the Tricuspid valve). The Tricuspid and Mitral results are quite good, and in agreement to other results published in the literature [2, 3].

\section{Conclusion}

This paper introduces an embedding subspace, which is generated by collecting delay vectors and using the entropy gradient as a predictability measure. In this subspace the heart sounds dynamics are assessed, and the results (in the different auscultation spots) show an increase in accuracy when compared to our previous results [4] and only using one topological feature ( $n$ orbits). We can conclude that the heart murmur trajectories in the embedding subspace are substantially different when compared to healthy patients. Given the presented results, we argue that the trajectories in the projected embedding subspace are a very promising avenue given the physiological clues that this new space seems to encode.

\section{Acknowledgements}

This article is a result of the project NanoSTIMA, NORTE-01-0145-FEDER-000016, supported by Norte Portugal Regional Operational Programme (NORTE 2020), through Portugal 2020 and the European Regional Development Fund.

\section{References}

[1] A.Guyton, J.E.Hall, eds., Textbook of Medical Physiology. Elsevier Saunders, 11th ed., Ed Hall, Jun 2006

[2] C. Ahlstrom, P. Hult, P. Rask, J. Karlsson, E. Nylander, U.Dahlstrom, P.Ask "Feature extraction for Systolic Heart Murmur Classification”, Ann Biomed Eng, vol. 34, no. 11, pp. 1666-1677, 2006.

[3] E. Delgado-Trejos et al., "Digital auscultation analysis for heart murmur classification,” Ann Biomed Eng, vol. 37, no. 2, pp. 337353, 2009.

[4] J.Oliveira, C.Oliveira, B.Cardoso, M.S.Sultan, M.Coimbra, "A multi-spot exploration of the topological structures of the reconstructed phase-space for the detection of cardiac murmurs", Proc. IEEE EMBC 2015.

[5] J.Oliveira, A.Castro, M.Coimbra, "Exploring Embedding Matrices and the Entropy Gradient for the Segmentation of Heart Sounds in Real Noisy Environments”, Proc. IEEE EMBC 2014.

[6] H. Kantz, T. Schreiber Nonlinear Time Series Analysis, 2th ed. Ed. Cambridge University Press, Jan 2004.

[7] D.Kumar, P.Carvalho, M.Antunes, R.P.Paiva, J.Henriques, "Heart murmur classification with feature selection.” IEEE EMBC 2010

[8] A. W. Bowman, and A. Azzalini, "Applied Smoothing Techniques for Data Analysis”. New York: Oxford University Press Inc., 1997.

[9] D. Pereira, F. Hedayioglu, R. Correia, T. Silva, I. Dutra, F. Almeida, S. S. Mattos, M. Coimbra, "DigiScope - Unobtrusive Collection and Annotating of Auscultations in Real Hospital Environments", IEEE EMBC 2011.

Address for correspondence.

Name. Jorge Oliveira

Address. Rua do Campo Alegre, 1021/1055 4169-007 Porto Portugal

E-mail address. oliveira_jorge@dcc.fc.up.pt 